\title{
Altered proteolytic events in experimental autoimmune encephalomyelitis discovered by iTRAQ shotgun proteomics analysis of spinal cord

\author{
Mohit Raja Jain ${ }^{\dagger 1}$, Shengjie Bian ${ }^{\dagger 1}$, Tong Liu ${ }^{\dagger 1}$, Jun Hu${ }^{1}$, Stella Elkabes ${ }^{2}$ and
} Hong Li*1
}

Address: ${ }^{1}$ Center for Advanced Proteomics Research and Department of Biochemistry and Molecular Biology, UMDNJ-New Jersey Medical School Cancer Research Center, Newark, NJ 07103, USA and 2Department of Neurology and Neuroscience, UMDNJ-New Jersey Medical School, Newark, NJ 07103, USA

Email: Mohit Raja Jain - jainmr@umdnj.edu; Shengjie Bian - biansh@umdnj.edu; Tong Liu - linto@umdnj.edu; Jun Hu - huju@umdnj.edu; Stella Elkabes - elkabest@umdnj.edu; Hong Li* - liho2@umdnj.edu

* Corresponding author †Equal contributors

Published: 16 July 2009

Proteome Science 2009, 7:25 doi:10.1186/1477-5956-7-25
Received: 4 February 2009

Accepted: 16 July 2009

This article is available from: http://www.proteomesci.com/content/7/I/25

(c) 2009 Jain et al; licensee BioMed Central Ltd.

This is an Open Access article distributed under the terms of the Creative Commons Attribution License (http://creativecommons.org/licenses/by/2.0), which permits unrestricted use, distribution, and reproduction in any medium, provided the original work is properly cited.

\begin{abstract}
Background: Abnormal activation of protease activities during experimental autoimmune encephalomyelitis (EAE) in rats, a rodent model of multiple sclerosis, have been implicated in either the direct destruction of myelin components or the intracellular signal transduction pathways that lead to lymphocyte infiltration, oligodendrocyte destruction, neuronal dysfunctions and axonal degeneration. The identification of changes in regulated proteolytic events during EAE is crucial for uncovering activated proteases that may underline the pathological features such as inflammation and demyelination. We searched for either non-tryptic or semi-tryptic peptides from a previous shotgun proteomics study using isobaric tags for relative and absolute quantification (iTRAQ) to compare the proteomes of normal and EAE rat lumbar spinal cords.
\end{abstract}

Results: We discovered that several proteins, such as $\alpha_{1}$-macroglobulin, a protease inhibitor, $\alpha_{1} B-$ glycoprotein, $\beta_{2}$-microglobulin, neurofilament light polypeptide and sulfated glycoprotein I had non-tryptic peptide iTRAQ ratios that were substantially different from the overall protein iTRAQ ratios, suggesting that such peptides may be markers for the proteolytic products generated by the protease(s) altered during EAE. Indeed, subsequent Western blotting confirmed the dysregulation of specific protein cleavages in EAE tissues. Additional proteolytic changes in $\alpha_{2}$-macroglobulin, another protease inhibitor similar to $\alpha_{1}$-macroglobulin was also observed.

Conclusion: The results from this study revealed changes among both neuronal protein processing and endogenous proteolysis modulators in EAE animals. This information may provide a rationale for protease inhibitor-based therapeutic interventions for multiple sclerosis.

\section{Background}

Proteases and peptidases are important regulators that govern many cellular functions [1]. Some protease activi- ties are manifested globally, e.g. during protein turnover in lysosomes and proteasomes. Other proteases are activated only within particular defined contexts, serving spe- 
cific signal transduction and other regulatory functions. Well-known examples include the caspase cascade during apoptosis, the coagulation cascade during clot formation and the classical and alternative complement innate immune systems for pathogen clearance. More recently, regulated proteolysis events have been implicated in numerous disease-related processes, including calpain activation following excitotoxicity in neuronal cells [2], matrix metalloprotease (MMP) modulation during cancer metastasis and multiple sclerosis [3], and the contribution of rennin and angiotensin converting enzyme to regulate blood pressure and cardiovascular function [4]. Understanding how protease activities are regulated is important both for discerning basic biological mechanisms and for developing therapies that can regulate pathological protease activities.

Quantitative proteomics techniques have been developed to uncover regulated proteolysis events (i.e. the identification of the cleavage sites within targeted proteins) and possibly identify the responsible protease(s). These methods can be broadly divided into following categories: 1 ) quantification of changes among selective proteases; 2 ) quantification of known protease substrates; 3 ) selective quantification of protein N-termini. For example, Cravatt et al. pioneered an activity-based proteomics technique to use selective inhibitors for affinity enrichment and quantification of proteases [5]. Overall et al. developed several strategies to quantify changes in known or putative protease substrates in cells and tissues expressing different levels of MMPs with great success [6,7]. In particular, their work has utilized the iTRAQ technique for the identification of protease substrates and determination of proteolytic sites [8]. In line with the general theme that subproteome analyses are usually more sensitive than global proteomic studies for specific biological objectives. Van Damme et al. refined a technique called combined fractional diagonal chromatography to separate N-terminal peptides from their internal tryptic counterparts for more sensitive degradomic analysis [9]. Recently, Wells group demonstrated an elegant technique for global identification of proteolytic cleavage sites within proteins in apoptotic cells by specific labeling of protein N-termini [10]. After, selectively biotinylating the $\alpha$-amines of $\mathrm{N}$-termini of peptides (including neo N-termini produced during apoptosis) with subtiligase, derivatized tryptic peptides were enriched on an avidin media, and the sites of proteolytic cleavage were identified by LC-MS/MS. All of these specialized methods and experimental designs have been proven effective for revealing different aspects of the degradome. Given the complexities involved in biological systems, however, it may not always be possible to predict, whether altered proteolytic events are important for a study in which proteomics analysis is needed. Because of this, there are many quantitative data that have already been obtained for expression proteomics studies could contain valuable information on altered proteolytic events that are critical for the underlying biological scenarios. In this report, we demonstrate a simple method to uncover regulated proteolytic events from a quantitative shotgun proteomics dataset obtained using the iTRAQ technique [11]. The validity of our current approach is based on the assumptions that: 1) regulated proteolytic activities are typically non-tryptic, so the resulting peptides would be either semi- or non-tryptic and 2) the iTRAQ expression ratios for these peptides are different from those of the tryptic peptides derived from the same protein. Obviously, these assumptions do not apply to the targets of those proteases whose substrate specificities overlap with trypsin.

The model systems used in the present study were the spinal cords isolated from rats suffering from EAE, a wellcharacterized animal model of multiple sclerosis [4,11]. This human neurodegenerative autoimmune disease is characterized by inflammation, demyelination in the central nervous system (CNS), axonal damage and neuronal loss leading to neurological deficits such as paresis and paralysis. Since the mechanisms underlying the damages to CNS cells in this disease remain elusive, we have previously utilized the iTRAQ technology to define differentially expressed and post-translationally modified proteins during acute EAE in the Lewis rat lumbar spinal cord, the region most affected in this model $[11,12]$. Elevated levels of proteases such as MMPs have previously been suggested to play a role in mediating EAE pathology [4], and functional inhibition of selective MMP activities has been shown to alleviate EAE symptoms [4]. Interestingly, inhibition of MMP-2 and MMP-12 made some animals more susceptible to EAE [13], suggesting that the proteolytic activity in EAE is rather complex and providing a rationale for further analysis of the EAE degradome. In this study, we were able to identify changes in regulated proteolysis events in EAE via searching for non-tryptic and semi-tryptic peptides. By comparing the iTRAQ expression ratios of the semi and non-tryptic fragments with their putative protein expression ratios averaged from the iTRAQ ratio of all tryptic peptide, we were able to uncover changes in selective CNS proteins and endogenous protease inhibitors. These results were in accord with known protease dysregulations reported in tissues of both EAE animals and multiple sclerosis patients. This additional information clearly complements differential expression data commonly sought after by those performing shotgun proteomics studies and it can be obtained with relatively little additional effort and cost, thus improving proteomic research productivity. 


\section{Results}

Identification of Differentially Cleaved Protein Products

In our previous study, we reported the identification of 41 differentially expressed proteins [11] and changes in citrullination, methylation and phosphorylation of various proteins [12] in the spinal cord of EAE animals. To our surprise, we also discovered the increased levels of a distinct proteolytic fragment ( $50 \mathrm{kDa})$ of moesin in EAE spinal cords, following Western blotting validation analysis of iTRAQ quantitation results [11], suggesting that there may be other proteolytic products present in EAE animal tissues. In the current study, we examined proteins that were differentially cleaved in EAE animals. We identified 197 semi-tryptic or non-tryptic peptides belonging to 104 proteins (see Additional file 1). Among these, we found seven proteolytic products whose levels were significantly altered in EAE compared to control animals, although the corresponding total protein levels were not changed to the same degrees (Table 1, see Additional files
2 and 3). For example, a semi-tryptic peptide $\left({ }^{437} \mathrm{GFCEVCK}^{443}\right)$ from sulfated glycoprotein 1 , produced from N-terminal non tryptic cleavage between G436 and G437, was found to be dramatically increased in EAE tissues and had an iTRAQ ratio of 4.5 (Fig. 1A). However, its total protein level deduced from all the tryptic peptides (see Additional file 2) observed for this protein was altered to a lesser extent (EAE/control ratio of 1.9, Table 1), as demonstrated by the iTRAQ reporter ion regions for a representative tryptic peptide ${ }^{68}$ TVVTEAGNLLK 78 for this protein (Table 1 and Fig. 1B). Similarly, a semi-tryptic peptide ${ }^{807}{ }^{6 L E L T L P Y S V V R} 818$ from $\alpha_{1}$-macroglobulin $\left(\alpha_{1} M\right)$ (Fig. 1C) was found to be significantly elevated with an ITRAQ ratio of 5.9 in the EAE tissues; however, the total $\alpha_{1}$ M protein level was increased with an ITRAQ ratio of 2.8 (Table 1 and Fig. 1D). Likewise, another semi-tryptic peptide ${ }^{84}$ ILAHTEFTPTETDVYACR ${ }^{101}$ from $\beta_{2}$ microgloublin was found to be increased with an iTRAQ ratio of 3.1 in the EAE tissue as compared to an iTRAQ

Table I: Altered non-tryptic cleaved peptides in EAE rat spinal cord

\begin{tabular}{|c|c|c|c|c|c|c|c|c|}
\hline Protein & $\begin{array}{l}\text { Swiss-Prot } \\
\text { Accession } \\
\text { Number }\end{array}$ & Peptide $^{a}$ & $\begin{array}{c}\text { Observed } \\
\text { Mass }(\mathrm{m} / \mathrm{z})\end{array}$ & Error (ppm) & $\begin{array}{c}\text { Peptide } \\
\text { Ratio }^{b} \pm \text { SD }\end{array}$ & p-Value & $\begin{array}{c}\text { Protein } \\
\text { Ratio (N) }\end{array}$ & $\begin{array}{c}\text { Sequence } \\
\text { Coverage } \\
(\%)\end{array}$ \\
\hline $\begin{array}{l}\alpha_{1} B- \\
\text { glycoprotein }\end{array}$ & $\frac{\text { Q9EPHI|AIB }}{\underline{G}}$ & $\begin{array}{l}\text { GPGNA- } \\
{ }^{2} \text { LWLDSGSEP } \\
\text { ELR }^{32}-A E P Q S\end{array}$ & 1545.743 & 38 & $4.3 \pm 1.2$ & 0.02 & $3.3(5)$ & 6 \\
\hline $\begin{array}{l}\alpha_{1^{-}} \\
\text {Macroglobulin }\end{array}$ & Q6304IIAIM & $\begin{array}{l}\text { VFQPF- } \\
\text { 807FLELTLPYS } \\
\text { VVR }^{818-G E A F I ~}\end{array}$ & 1580.917 & 0 & $5.9 \pm 1.6$ & 0.03 & $2.8(19)$ & 13 \\
\hline $\begin{array}{l}\beta_{2^{-}} \\
\text {Microglobulin }\end{array}$ & $\underline{P 07|5| \mid B 2 M G}$ & $\begin{array}{l}\text { DWSFY- } \\
\text { 83|LAHTEFTPT } \\
\text { ETDVYACR I01 } \\
\text {-VKHVT }\end{array}$ & 2257.067 & 4 & $3.1 \pm 0.8$ & 0.02 & $2.7(2)$ & 26 \\
\hline $\begin{array}{l}\text { Neurofilament } \\
\text { light } \\
\text { polypeptide }\end{array}$ & $\underline{\text { PI9527|NFL }}$ & $\begin{array}{l}\text { AFPAY- } \\
\text { 443YTSHVQEE } \\
\text { QSEVEETIEAT } \\
\text { K } 463 \text {-AEEAK }\end{array}$ & 2625.292 & 6 & $2.2 \pm 0.6$ & 0.05 & $0.9(75)$ & 56 \\
\hline $\begin{array}{l}\text { Phospho } \\
\text { glucomutase-I }\end{array}$ & P38652/PGMI & $\begin{array}{l}\text { KFKPF- } \\
\text { 184TVEIVDSVE } \\
\text { AYATMLR200_ } \\
\text { NIFDF }\end{array}$ & 1941.006 & -3 & $1.6 \pm 0.1$ & 0.01 & I.I (6) & 17 \\
\hline $\begin{array}{l}\text { Sulfated } \\
\text { glycoprotein I }\end{array}$ & PI0960|SAP & $\begin{array}{l}\text { QPKAN- } \\
\text { 194EDVCQDC } \\
\text { MK'202-LVTDI }\end{array}$ & 1450.59 & 11 & $2.4 \pm 0.2$ & 0.00 & $1.9(16)$ & 19 \\
\hline $\begin{array}{l}\text { Sulfated } \\
\text { glycoprotein I }\end{array}$ & PI0960|SAP & $\begin{array}{l}\text { PQKNG- } \\
{ }^{437} \text { GFCEVCK } 44 \\
{ }^{3}-K L V I Y\end{array}$ & II 65.508 & -3 & $4.5 \pm 0.7$ & 0.00 & $1.9(16)$ & 19 \\
\hline
\end{tabular}

a Sequences bracketed by amino acid numbers are the semi-tryptic peptides discovered experimentally. Five amino acids before and after the semitryptic peptides are shown to indicate the non tryptic cleavage sites.

b iTRAQ Ratio: EAE/Control

c Total number of peptides identified for respective protein (see Additional file 2) 


\section{Semi-tryptic peptides}

\section{Sulfated glycoprotein 1}

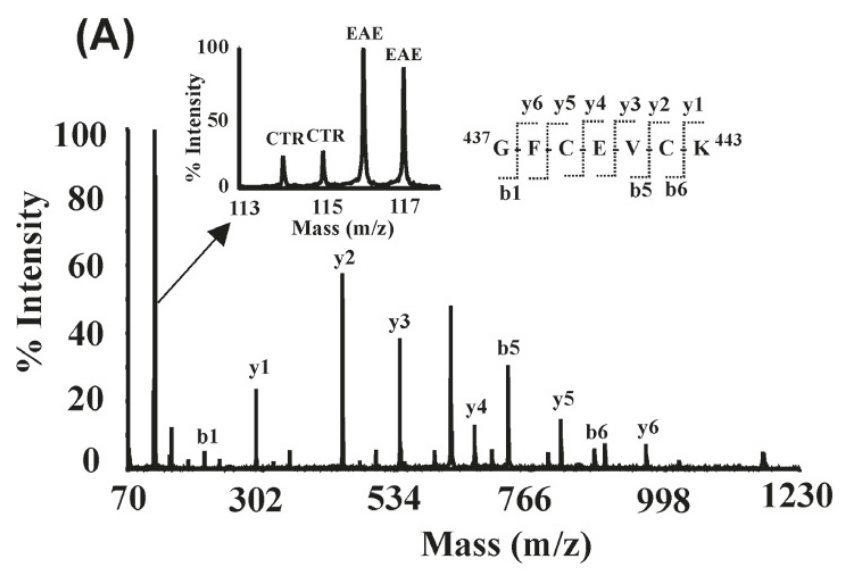

$\alpha_{1}$-macroglobulin

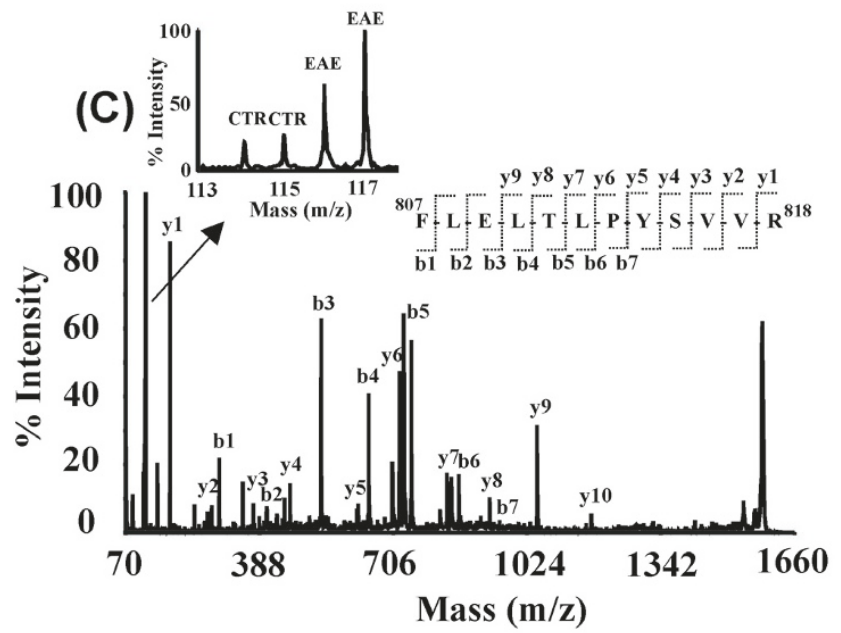

\section{Tryptic peptides}
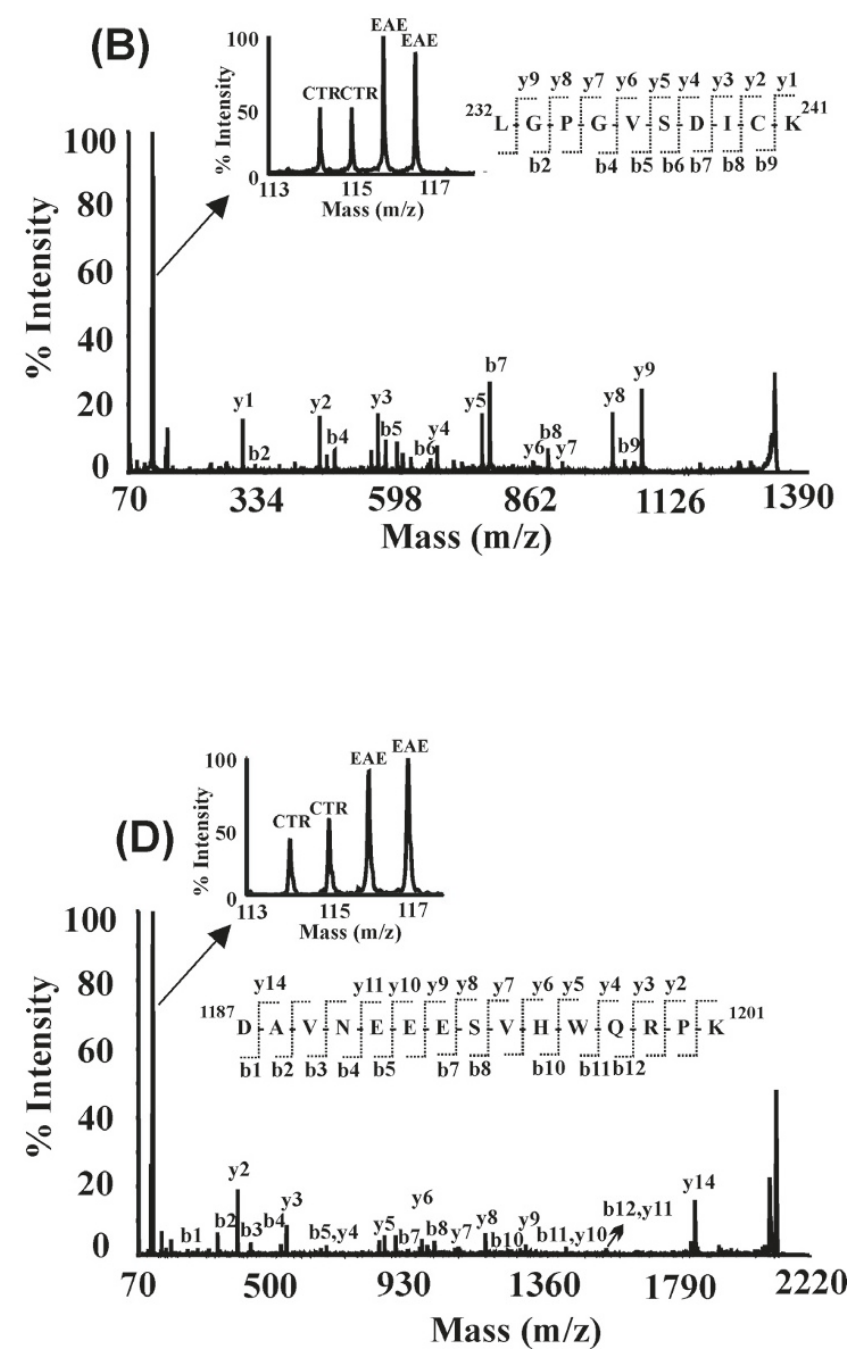

Figure I

MS/MS spectra of representative semi-tryptic and tryptic peptides. iTRAQ reporter ion region and peptide sequencing region of the MS/MS spectrum for semitryptic peptides derived from (A) sulfated glycoprotein I (437-443) and (C) $\alpha_{1}$-macroglobulin (807-8I8). Representative tryptic peptides changes are shown for (B) sulfated glycoprotein I(232-24I) and (D) $\alpha_{1}$ macroglobulin (I I87-I20I). Peptide sequences were deduced from the MS/MS spectra based on the observation of continuous series of either $\mathrm{N}$-terminal (b-series) or $\mathrm{C}$-terminal ( $\mathrm{y}$-series) ions. The peak areas of iTRAQ quantification (shown in insets of $A, B, C, D)$ ions, $\mathrm{m} / \mathrm{z}$ II4-II7 were used to measure the relative abundance of individual peptides.

ratio of 2.7 in total $\beta_{2}$-microgloublin protein level (Table 1). Interestingly, the semi-tryptic peptides from both neurofilament light

polypeptide (443YTSHVQEEQSEVEETIEATK ${ }^{463}$ ) and phosphoglucomutase-1 (184TVEIVDSVEAYATMLR ${ }^{200}$ ) were higher (iTRAQ ratios of 2.2 and 1.6 respectively) in EAE tissues. However, no significant changes were observed in their protein levels (iTRAQ ratios of 0.9 and 1.1 respectively, Table 1) in EAE tissues.

\section{Corroboration of ITRAQ analysis by Western Blot}

In this study, we observed that several proteins previously implicated in EAE etiology were differentially processed. A highly specific antibody for rat $\alpha_{1} M$ is not commercially available for validation. However, rat $\alpha$-macroglobulins 1 and $2\left(\alpha_{2} \mathrm{M}\right)$ are similar in their amino acid sequence (56\% of sequence homology, see Additional file 4) and have been suggested to have comparable three-dimensional structures and function [14]. We tested whether 
$\alpha_{2} \mathrm{M}$ may also be similarly processed as with $\alpha_{1} \mathrm{M}$ in EAE by analyzing the presence of both breakdown products using an antibody against $\alpha_{2} \mathrm{M}$ (Fig. 2A). Interestingly, the levels of both intact $\alpha_{2} \mathrm{M}(\sim 165 \mathrm{kDa})$ and a proteolytic product $(\sim 80 \mathrm{kDa})$ were elevated in the EAE tissues than the controls (Fig. 2A). Another $\alpha_{2} \mathrm{M}$ proteolytic product $(\sim 60 \mathrm{kDa})$ levels were also found to be increased in the EAE tissues (Fig. 2A). Using a longer exposure of the Western blotting film, more proteolytic fragments can be observed in the EAE tissues (see Additional file 5). We further probed for the presence of proteolytic products from other proteins for which the specific antibodies are commercially available. In the case of $\beta_{2}$-microglobulin, which was increased in the EAE tissues (iTRAQ ratio of 2.7, Table 1), we found that the levels of both the intact protein as well as its proteolytic fragment were dramatically increased in the EAE tissues by Western blotting (Fig. $2 \mathrm{~B})$, corroborating the observations made from the iTRAQ experiment. We also probed for the presence of proteolytic products of sulfated glycoprotein 1 . The levels of the intact protein $(\sim 62 \mathrm{kDa})$ were found to be increased in the EAE tissues (see Additional file 5), corroborating iTRAQ results (Table 1). However, we could not find the presence of its proteolytic products, which may be indicative of either rapid degradation of the proteolytic products or changes in the epitopes for the antibody used. The neurofilament light polypeptide was found unchanged in the EAE tissues by iTRAQ analysis (Table 1). Using an antineurofilament light polypeptide antibody, we confirmed that intact neurofilament light polypeptide ( $\sim 68 \mathrm{kDa})$ was not changed in the EAE tissues (see Additional file 5); however, by comparison, a putative proteolytic product ( $\sim 11 \mathrm{kDa}$ ) was increased by 10\% in the EAE tissues (see Additional file 5).

\section{Discussion}

There are many techniques for the analysis of the degradome. Although the methods employed here do not incorporate enrichment techniques, we were able to identify cleaved proteins. Although we assumed only nontryptic fragments as markers for cleaved proteins in the spinal cord of rats affected by EAE, there are proteases especially in the serine protease family - whose substrate cleavage specificities may overlap with trypsin. In principle, such cleavages may also be identified by iTRAQ analysis, but it is difficult to distinguish endogenous cleavages from tryptic cleavages of extracted proteins. Under such circumstances, the techniques developed by McDonald $e t$ al [15] and Ji et al [16] to first block the protein N-termini prior to tryptic digestion and enrichment of the neo-N-termini should be used to discover the proteolytically modulated proteins. Overall, we have observed nearly 200 non or semi-tryptic events in over 100 proteins. It is likely that significantly more such events could be detected with focused sub-proteomic analysis of the degradomes, using the approaches elegantly described by Wells [10], Van Damme [9] and others. However, since most expression proteomics experiments have been conducted without the
$\begin{array}{llllll}\mathrm{C} 1 & \mathrm{C} 2 & \mathrm{C} 3 & \mathrm{E} 1 & \mathrm{E} 2 & \mathrm{E} 3\end{array}$

(A)

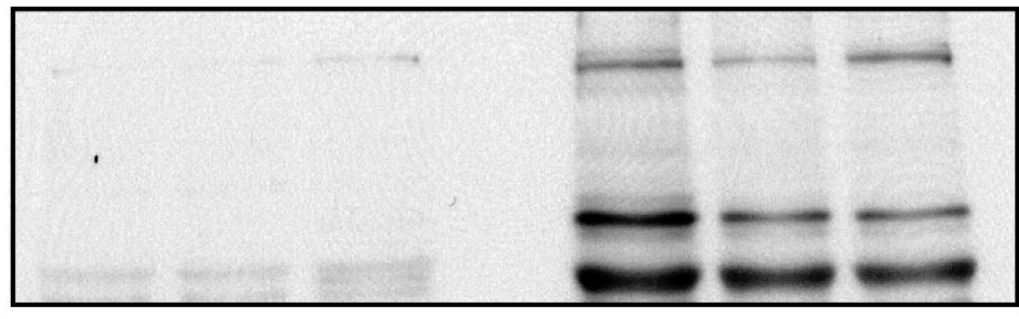

(B)

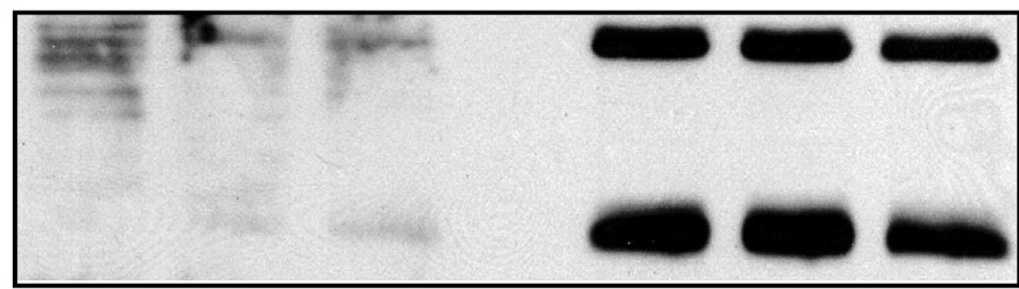

(C)

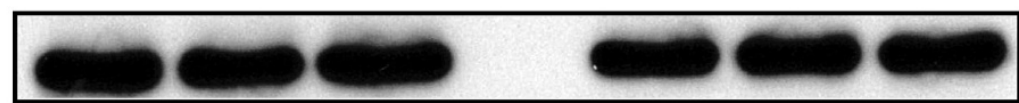

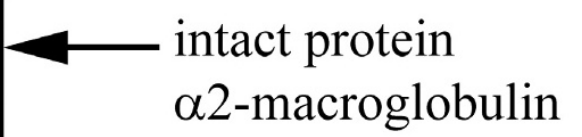

a2-macroglobulin

proteolytic fragment ( $\sim 80 \mathrm{kDa} \& \sim 60 \mathrm{kDa})$

intact protein $\beta 2$-microglobulin

proteolytic fragment $(\sim 9 \mathrm{kDa})$ $\underline{\text { GAPDH }}$

Figure 2

Western blot validation of select protein cleavages. (A) $\alpha_{2}$-macroglobulin, (B) $\beta_{2}$-microglobulin from both control (CI, $\mathrm{C2}, \mathrm{C} 3$ ) and EAE (EI, E2, E3) animals. (C) GAPDH was used to determine the equal loading of proteins for all the samples. 
enrichment of the protein N-termini, it is important to be able to extract differential proteolytic signals from such studies in addition to protein expression information, considering the significant amount of resources devoted for such experiments. We have demonstrated here that it is possible to obtain such information with bioinformatic data reprocessing. The existence of differential proteolysis in the expression proteomic datasets may provide a rationale for subsequently more focused degradomic studies.

EAE is an established animal model for studying cellular pathways leading to demyelination, axonal damage, lymphocyte infiltration of the CNS, and other processes that occur in CNS of multiple sclerosis patients [17-19]. Our previous iTRAQ analysis of the spinal cord in acute EAE revealed 41 differentially expressed proteins, including complement C3, $\alpha_{2} \mathrm{M}$, ceruloplasmin, and other acute phase proteins commonly associated with systematic inflammation [11]. During different stages of multiple sclerosis and EAE, aberrant proteolytic processes have been reported to be involved in axonal damage, oligodendrocyte apoptosis, demyelination, pro-inflammatory cytokine activation, epitope spreading, $\mathrm{T}$ cell and macrophage activation, and damage to the blood brain barrier [4]. Although some of these proteolytic events involve tissue triage and broader damage, under most circumstances, the implicated proteolytic events involve limited cleavages at specific peptide bonds [4], suggesting the existence of regulated proteolytic events. The dysregulation of proteolysis in EAE pathology may be dependent on protease levels, localization, activation and endogenous inhibitor concentrations [4]. For example, MMPs including MMP-9, 2, 14, 3, 7, 12, 8 have been reported to be elevated at different stages of multiple sclerosis [4,13], and they are important promoters of immune cell extravasation into the CNS via the opening of the blood brain barrier due to their unique ability to facilitate fibronolysis [20]. In addition to MMPs and their inhibitors, serine proteases, such as tissue plasminogen activator (tPA), urokinase plasminogen activator, thrombin, elastase, tissue kallikreins and their inhibitors have also been reported to be activated in various multiple sclerosis patients and animal models [4].

Among the proteins that were found to have increased proteolytic cleavage products is $\alpha_{1} \mathrm{M}$, also known as pregnancy zone protein. It is a widely expressed plasma glycoprotein protease inhibitor that belongs to the $\alpha_{2} \mathrm{M}$ family [21]. These protease inhibitors are synthesized in the liver and form dimeric and tetrameric complexes in vivo. $\alpha_{1} \mathrm{M}$ has been shown to be cleaved by both MMP-2 and MMP9 , and it is capable of inhibiting mast cell tryptase, tPA, chymotrypsin and snake venom metalloprotease $[22,23]$. These protease inhibitors function by forming thiol esters with protease side chains following their cleavage by the proteases with a "bait region" and inhibiting protease activity towards other high molecular weight substrates [24]. $\alpha_{1} M$ has been shown to selectively inhibit T-cell activation and IL-2 secretion [25] and also binds to both TGF$\beta 1$ and TGF- $\beta 2$ [26], inhibiting their association with their cell surface receptors, which may be protective for EAE animals [25]. Given the functional significance of $\alpha_{1} M$, the observation of the increase of its cleavage products in EAE suggests that it may play a protective role by dampening the effect of harmful proteases. Similarly, $\alpha_{2} M$ has been shown by Western blot analysis to be cleaved (increase in an $80 \mathrm{kDa}$ fragment) in EAE spinal cords. Our previous studies have shown a significant increase (iTRAQ ratio of 2.3) in $\alpha_{2} \mathrm{M}$ protein levels [11]. $\alpha_{2} \mathrm{M}$ can be produced by activated macrophages, has been shown to attenuate EAE symptoms when administered exogenously, and may exert its beneficial effects by either neutralizing proteinases involved in tissue damage or directly interfering with antigen recognition due to its ability to bind myelin basic protein [24]. EAE is an inflammatory autoimmune condition in affected animals. It is interesting to see that several immune system proteins were differentially cleaved in EAE. $\beta_{2}$-microglobulin is part of MHC class I molecule that is important for antigen presentation [27]. Neurofilament light polypeptide has been reported to be degraded in EAE animals putatively by calpain [28] and possibly the result of increased oxidative stress during EAE [29]. Based on our proteomics study, it appears that proteins related to neuroinflammation, neuroregeneration and axonal integrity may proteolytically processed in EAE. Further studies are needed to determine the functional significance of these cleaved proteins.

\section{Conclusion}

Proteolysis is an important means of post-translational regulation of neuronal cell function; its dysregulation may underlie the pathology of EAE and multiple sclerosis. Many of the implicated proteases are important regulator of cytokines and chemokines [4]. Changes in different protease inhibitors discovered in this study, like $\alpha_{1} M$ and $\alpha_{2} \mathrm{M}$ (Table 1) and their proteolytic fragments have been reported in recent proteomics searches for clinical biomarkers. For example, a cleaved product of cystatin C, an inhibitor of cysteine proteases has been reported as a potential biomarker in the cerebrospinal fluid of multiple sclerosis patients [30], although the validity of this peptide as disease biomarker has recently been challenged by another study [31]. The results from our current study revealed changes among both neuronal protein processing and endogenous proteolysis modulators. This information may provide a rationale for further studies to develop protease inhibitor-based therapeutic interventions for demyelinating diseases and multiple sclerosis. 


\section{Methods \\ Induction of EAE}

Two month-old Lewis rats were immunized with myelin basic protein (MBP) emulsified in complete Freund's adjuvant (CFA) or CFA-containing vehicle according to Nicot et al [32]. Rats were maintained in a standard $12 \mathrm{~h}$ light/dark cycle and had free access to water and food based on approved IACUC protocols. EAE clinical symptoms were scored as follows: 1 , tail weakness; 2 , hind limb weakness; 3, hind limb paralysis; 4, quadriplegia; and 5, moribund. For this study, lumbar spinal cords (region most affected by EAE) were harvested when EAE-induced rats exhibited hind limb paralysis (clinical score 3 ). The dissected lumbar spinal cords were immediately frozen on dry ice and stored at $-80^{\circ} \mathrm{C}$ until further use.

\section{Protein extraction and iTRAQ labeling}

For iTRAQ analysis, detailed methods have been described previously [11]. Briefly, lumbar spinal cords obtained from two CFA-treated controls and two rats affected by EAE were used. Fifteen milligrams of the spinal cord tissues were homogenized in $300 \mu \mathrm{l}$ of a lysis buffer consisting of $25 \mathrm{mM}$ triethylammonium bicarbonate, 20 $\mathrm{mM}$ sodium carbonate and $2 \mu \mathrm{l}$ of protease inhibitor cocktail (Sigma, St Louis, MO, USA). The supernatant was cleared by centrifugation at $19,000 \times \mathrm{g}$ for $30 \mathrm{~min}$, and the $\mathrm{pH}$ was adjusted to 8.0 with $0.1 \mathrm{M}$ HEPES. The iTRAQ labeling procedures were performed according to the manufacturer's instructions (Applied Biosystems (ABI), Foster City, CA). Ninety micrograms of soluble proteins from each sample was reduced by the addition of $2 \mu \mathrm{l}$ of the reducing agent, tris (2-carboxyethyl) phosphine hydrochloride (TCEP) and incubated at $60^{\circ} \mathrm{C}$ for $1 \mathrm{~h}$. Reduced cysteines were then alkylated with the addition of $1 \mu \mathrm{l}$ of $200 \mathrm{mM}$ methyl methanethiosulfonate (MMTS) and incubated at room temperature for $10 \mathrm{~min}$. To initiate tryptic digestion, $10 \mu \mathrm{g}$ of trypsin (Promega Corporation, Madison, WI USA) was added to each of the four samples and incubated at $37^{\circ} \mathrm{C}$ overnight. The resulting peptides were labeled with the appropriate iTRAQ reagents. Samples derived from two different control spinal cords were labeled with iTRAQ tags 114 and 115, whereas samples obtained from two independent EAE spinal cords were labeled with tags 116 and 117 . The labeled samples were then mixed together and fractionated via a strong cation exchange chromatography (SCX) on a BioCAD ${ }^{\text {тм }}$ Perfusion Chromatography System (ABI), equipped with a polysulfoethyl A column $(4.6 \mathrm{~mm} \times 200 \mathrm{~mm}, 5 \mu \mathrm{m}, 300$ $\mathrm{A}^{\circ}$, Poly LC Inc., Columbia, MD, USA) and an upstream guard column $(4.0 \mathrm{~mm} \times 10 \mathrm{~mm}$, Poly LC). The peptide mixture was separated with a gradient consisting of mobile phase A, containing $10 \mathrm{mM} \mathrm{KH}_{2} \mathrm{PO}_{4}$ and $20 \%$ acetonitrile (ACN) ( $\mathrm{pH} 3.0)$, and mobile phase $\mathrm{B}$, consisting of $600 \mathrm{mM} \mathrm{KCl}, 10 \mathrm{mM} \mathrm{KH}_{2} \mathrm{PO}_{4}$ and 20\% ACN (pH 3.0). Labeled peptides were eluted with a 40-min linear gradi- ent from 0 to $50 \% \mathrm{~B}$, followed by another $10 \mathrm{~min}$ from 50 to $100 \% \mathrm{~B}$. Two-minute fractions were dried via speed-vac and desalted via PepClean ${ }^{\mathrm{TM}} \mathrm{C}_{18}$ spin columns (Pierce, Rockford, IL, USA). Desalted peptides were further fractionated on an Ultimate ${ }^{\mathrm{TM}}$ Chromatography System equipped with a Probot matrix-assisted laser desorption ionization (MALDI) spotting device (Dionex, Sunnyvale, CA, USA). Peptides were first captured onto a reversed phase trapping column $(0.3 \mathrm{~mm} \times 5.0 \mathrm{~mm})$ and then resolved on a $0.1 \mathrm{~mm} \times 150 \mathrm{~mm}$ capillary PepMap column ( $3 \mathrm{um}, 100 \mathrm{~A}^{\circ}, \mathrm{C}_{18}$, Dionex), with a 70 -min gradient of solvent A ( $5 \%$ ACN, $0.1 \%$ trifluoroacetic acid, TFA) and solvent B (95\% CAN and 0.1\% TFA): 0-4 min, from 5 to $8 \% \mathrm{~B}$; at $34 \mathrm{~min}$, to $18 \% \mathrm{~B}$; at $57 \mathrm{~min}$, to $35 \% \mathrm{~B}$ and at 64 $\min$, to $95 \% \mathrm{~B}$. The HPLC eluent was mixed in a $1: 3$ ratio with a MALDI matrix solution $(7 \mathrm{mg} / \mathrm{ml}$ alpha-cyano-4hydroxycinnamic acid, in $60 \% \mathrm{ACN}, 5 \mathrm{mM}$ of ammonium monobasic phosphate and the internal mass calibrants, $50 \mathrm{fmol} / \mu \mathrm{l}$ each of GFP and ACTH, 18-39) through a $30 \mathrm{nl}$ mixing tee and spotted onto the MALDI plates in an $18 \times 18$ spot array format. The peptides were analyzed on a 4700 Proteomics Analyzer MALDI-TOFTOF tandem mass spectrometer (ABI) in a data-dependent fashion using a job-wide interpretation method. MS spectra $(\mathrm{m} / \mathrm{z}$ 800-3,600) were acquired in positive ion reflector mode with internal mass calibration. A maximum of the ten most intense ions $(\mathrm{S} / \mathrm{N}>50)$ per spot were selected for subsequent MS/MS analysis in $1 \mathrm{k} \mathrm{eV}$ mode. Each spectrum was averaged over 4,000 laser shots.

\section{Protein database search and bioinformatics}

For automated peptide identification, ProteinPilot software (v. 2.0.1, Revision 67128, ABI) was used to process the tandem mass spectra to generate the peak lists for database search, using default parameters optimized by the manufacturer. For peptide identification, ProteinPilot uses both MS and MS/MS mass error tolerance based on established mass accuracy performance of the 4700 Proteomics Analyzer MALDI-TOF-TOF tandem mass spectrometer (ABI). The peak list was submitted for a "thorough" search against the rat sequences in the UniProtKB/Swiss-Prot v54.8 database (Release date Feb 05, 2008, 349,480 sequence entries) using the Paragon algorithm [33] with default parameters. The following search parameters were used: trypsin as a digesting agent, iTRAQlabeled N-termini and lysines and MMTS-labeled cysteines were set as fixed modifications; oxidized methionines and iTRAQ-labeled tyrosines were set as variable modifications. The Paragon algorithm automatically search for semi- and non-tryptic peptides in addition to tryptic ones. The semi-tryptic peptides identified with confidence interval (CI) values $\geq 99 \%$ and MS measurement error $\leq 50$ ppm were used for the degradome analysis. To reduce the probability of false identification, we chose to report only proteins containing at least two pep- 
tides with confidence values $\geq 95 \%$. Semi-tryptic peptides were also verified by manual examination of the spectra (see Additional file 3). The false discovery rate (FDR) was estimated by researching the spectra against a target decoy database containing both forward and reverse rat protein entries [34]. The FDR for this study was estimated to be $5.0 \%$. Relative quantification of peptides in each sample was calculated from the areas under the peaks at $\mathrm{m} / \mathrm{z}$ $114.1,115.1,116.1$ and 117.1. The calculated peak area ratios were corrected for overlapping isotopic contributions as per manufacturer's instructions. If peptides identified are common to different isoforms of related proteins, Pro Group ${ }^{\mathrm{TM}}$ Algorithm (ABI), a component within the ProteinPilot software, was used to calculate protein ratios using only iTRAQ ratios from the peptides that were distinct to each isoform and thus provide isoform-specific quantitation. P-values were derived via the 1-tailed Student's t-test for each peptide by comparing the two EAE with the two control values using Excel (Microsoft Corporation, Redmond, WA).

\section{Western blotting analysis}

Three independent control and EAE samples were used for Western blotting analysis. Thirty micrograms of proteins from each sample were first resolved on a $12.5 \%$ SDSPAGE gel and then transferred to a nitrocellulose membrane (Bio-Rad, Hercules, CA, USA). The membrane was rinsed with $\mathrm{PBS}$ and the non specific binding sites were blocked in a solution of 5\% nonfat milk in PBST $(0.05 \%$ Tween 20 in PBS) for $1 \mathrm{~h}$ at room temperature, followed by three washes in PBST for $10 \mathrm{~min}$ each. The membrane was first incubated with rabbit $\alpha_{2}-\mathrm{M}$ antibody (ab58703, Abcam Inc. Cambridge, MA, USA) (1:1,000 dilution) overnight and then washed in PBST buffer as described above. The immunocomplexes were visualized by Western Lightning ${ }^{\circledR}$ Western Blot Chemiluminescence Reagent Plus (PerkinElmer, Waltham, MA, USA), using goat antirabbit IgG coupled to horseradish peroxidase as the secondary antibody (170-6515, Bio-Rad, Hercules, CA, USA). The membrane was stripped and re-probed with either mouse anti-glyceraldehyde-3-phosphate dehydrogenase (10R-G109A, Fitzgerald, Concord, MA, USA) (1:10,000 dilution), or anti-neurofilament light polypeptide (sc-12980, Santa cruz biotechnology, Santa Cruz, CA, USA) (1:500 dilution), or anti- $\beta_{2}$-microgloblulin (sc69963, Santa cruz biotechnology, Santa Cruz, CA, USA) (1:500 dilution) and or anti-sulfated glycoprotein 1 (ab68466, Abcam Inc. Cambridge, MA, USA) $(1: 1,000$ dilution).

\section{Abbreviations}

EAE: experimental autoimmune encephalomyelitis; iTRAQ: isobaric tags for relative and absolute quantification.

\section{Competing interests}

The authors declare that they have no competing interests.

\section{Authors' contributions}

MRJ, SB, TL have performed the mass spectrometry, Western blot, statistical analysis and manuscript draft preparation. JH performed the bioinformatics. SE performed the EAE induction in animals, participated in discussion and manuscript preparation. HL conceived, designed the study and drafted the manuscript. All authors edited the manuscript and approved the final version.

\section{Additional material}

\section{Additional file 1}

List of identified peptides with semi-tryptic cleavages. ProteinPilot software (ABI) was used to process the tandem mass spectra and generate peak lists for the database search. Peptides identified with confidence interval values $\geq 99 \%$ are reported here.

Click here for file

[http://www.biomedcentral.com/content/supplementary/14775956-7-25-S1.xls]

\section{Additional file 2}

List of all the peptides identified for the proteins listed in Table 1. ProteinPilot software (ABI) was used to process the tandem mass spectra and generate peak lists for the database search. Peptides identified with the confidence interval values $\geq 95 \%$ are reported here and were used for calculation of the protein expression ratios.

Click here for file

[http://www.biomedcentral.com/content/supplementary/14775956-7-25-S2.xls]

\section{Additional file 3}

MS/MS spectra of the semi-tryptic peptides listed in Table 1. All MS/ MS spectra were acquired on a 4700 MALDI TOF/TOF tandem MS instrument (ABI). The spectra were matched to proteins by ProteinPilot software $(A B I)$.

Click here for file

[http://www.biomedcentral.com/content/supplementary/14775956-7-25-S3.pdf]

\section{Additional file 4}

Amino acid sequence alignment of the $\alpha_{1}$ and $\alpha_{2}$ macroglobulins. The alignment was performed using ClustalW. Rat $\alpha_{1}$ and $\alpha_{2}$ macroglobulins showing $~ 56 \%$ of sequence homology.

Click here for file

[http://www.biomedcentral.com/content/supplementary/14775956-7-25-S4.pdf] 


\section{Additional file 5}

Western blotting analyses. (A) Longer exposure image of $\alpha_{2}$-macroglobulin as shown in Fig. 2A. Low levels of the $\alpha_{2}$-macroglobulin fragments were present in the control spinal cords. More fragments can be seen in EAE samples (B) Sulfated glycoprotein 1 from both control (C1, C2, C3) and EAE (E1, E2, E3) animals. (C) Neurofilament light polypeptide Western blotting and (D) densitometry quantification of neurofilament light protein fragments signals. Quantification was performed with Quantity One software (Biorad) and the p-value was calculated using Excel (Microsoft). GAPDH was used to determine the equal loading of proteins for all the samples.

Click here for file

[http://www.biomedcentral.com/content/supplementary/14775956-7-25-S5.tiff]

\section{Acknowledgements}

The project was supported by NIH grants P30NS046593 (Neuroproteomics Core Grant) to H. L. and NS046363 to S.E. from the National Institute of Neurological Disorder and Stroke. The consent is solely the responsibility of authors and does not necessarily represent the official views of the National Institute of Neurological Disorders and Stroke or the National Institutes of Health.

\section{References}

I. Barrett A, Rawlings N, Woessner JF, Eds: Handbook of Proteolytic Enzymes. Second edition. San Diego: Academic Press; 2003.

2. Schaecher K, Rocchini A, Dinkins J, Matzelle DD, Banik NL: Calpain expression and infiltration of activated $T$ cells in experimental allergic encephalomyelitis over time: increased calpain activity begins with onset of disease. J Neuroimmunol 2002, 129:1-9.

3. Sternlicht MD, Werb Z: How matrix metalloproteinases regulate cell behavior. Annu Rev Cell Dev Biol 200I, 17:463-5I6.

4. Scarisbrick IA: The multiple sclerosis degradome: enzymatic cascades in development and progression of central nervous system inflammatory disease. Curr Top Microbiol Immunol 2008, 31 8: 133-175.

5. Cravatt BF, Sorensen E): Chemical strategies for the global analysis of protein function. Curr Opin Chem Biol 2000, 4:663-668.

6. Tam EM, Morrison CJ, Wu YI, Stack MS, Overall CM: Membrane protease proteomics: Isotope-coded affinity tag MS identification of undescribed MTI-matrix metalloproteinase substrates. Proc Natl Acad Sci USA 2004, 10 1:6917-6922.

7. Butler GS, Overall CM: Proteomic validation of protease drug targets: pharmacoproteomics of matrix metalloproteinase inhibitor drugs using isotope-coded affinity tag labelling and tandem mass spectrometry. Curr Pharm Des 2007, 13:263-270.

8. Dean RA, Overall CM: Proteomics discovery of metalloproteinase substrates in the cellular context by ITRAQ labeling reveals a diverse MMP-2 substrate degradome. Mol Cell Proteomics 2007, 6:61I-623.

9. Van Damme P, Martens L, Van Damme J, Hugelier K, Staes A, Vandekerckhove J, Gevaert K: Caspase-specific and nonspecific in vivo protein processing during Fas-induced apoptosis. Nat Methods 2005, 2:77I-777.

10. Mahrus S, Trinidad JC, Barkan DT, Sali A, Burlingame AL, Wells JA: Global sequencing of proteolytic cleavage sites in apoptosis by specific labeling of protein $\mathbf{N}$ termini. Cell 2008, 134:866-876.

II. Liu T, Donahue KC, Hu J, Kurnellas MP, Grant JE, Li H, Elkabes S: Identification of differentially expressed proteins in experimental autoimmune encephalomyelitis (EAE) by proteomic analysis of the spinal cord. J Proteome Res 2007, 6:2565-2575.

12. Grant JE, Hu J, Liu T, Jain MR, Elkabes S, Li H: Post-translational modifications in the rat lumbar spinal cord in experimental autoimmune encephalomyelitis. J Proteome Res 2007 6:2786-2791.
13. Weaver A, Goncalves da Silva A, Nuttall RK, Edwards DR, Shapiro $\mathrm{SD}$, Rivest S, Yong VW: An elevated matrix metalloproteinase (MMP) in an animal model of multiple sclerosis is protective by affecting Th I/Th2 polarization. Faseb J 2005, I9:1668-1670.

14. Sottrup-Jensen L: $\alpha$-macroglobulins: structure, shape, and mechanism of proteinase complex formation. I Biol Chem 1989, 264: II539-II542.

15. McDonald L, Robertson DH, Hurst JL, Beynon RJ: Positional proteomics: selective recovery and analysis of $\mathrm{N}$-terminal proteolytic peptides. Nat Methods 2005, 2:955-957.

16. Ji C, Guo N, Li L: Differential dimethyl labeling of $\mathbf{N}$-termini of peptides after guanidination for proteome analysis. J Proteome Res 2005, 4:2099-2108.

17. Steinman L: Assessment of animal models for MS and demyelinating disease in the design of rational therapy. Neuron 1999 , 24:5I|-5|4

18. Goes A van der, Dijkstra CD: Models for demyelination. Prog Brain Res 200I, 132:149-163.

19. Wekerle H, Kojima K, Lannes-Vieira J, Lassmann H, Linington C: Animal models. Ann Neurol 1994, 36(Suppl):S47-53.

20. Rosenberg GA: Matrix metalloproteinases and neuroinflammation in multiple sclerosis. Neuroscientist 2002, 8:586-595.

21. Saidi N, Samel M, Siigur J, Jensen PE: Lebetase, an $\alpha(\beta)$ fibrin(ogen)olytic metalloproteinase of Vipera lebetina snake venom, is inhibited by human $\alpha$-macroglobulins. Biochim Biophys Acta 1999, 1434:94-102.

22. Carlsson-Bosted L, Moestrup SK, Gliemann J, Sottrup-Jensen L, Stigbrand $\mathrm{T}$ : Three different conformational states of pregnancy zone protein identified by monoclonal antibodies. J Biol Chem |988, 263:6738-674|.

23. Sanchez MC, Chiabrando GA, Guglielmone HA, Bonacci GR, Rabinovich GA, Vides MA: Interaction of human tissue plasminogen activator (t-PA) with pregnancy zone protein: a comparative study with t-PA- $\alpha$. J Biochem 1998, I 24:274-279.

24. Petersen CM: $\alpha_{2}$-macroglobulin and pregnancy zone protein. Serum levels, $\alpha_{2}$-macroglobulin receptors, cellular synthesis and aspects of function in relation to immunology. Dan Med Bull 1993, 40:409-446.

25. Saito S, Hashimoto H, Yonemasu K, Ichijo M: Pregnancy zone protein inhibits production of interleukin-2 but does not affect interleukin-2 receptor expression on $\mathbf{T}$ cell activation. J Reprod Immunol 1990, I 7: I I 5-126.

26. Philip A, Bostedt L, Stigbrand T, O'Connor-McCourt MD: Binding of transforming growth factor- $\beta$ (TGF- $\beta$ ) to pregnancy zone protein (PZP). Comparison to the TGF- $\beta-\alpha_{2}$-macroglobulin interaction. Eur J Biochem 1994, 221:687-693.

27. Cresswell P, Ackerman AL, Giodini A, Peaper DR, Wearsch PA: Mechanisms of MHC class I-restricted antigen processing and cross-presentation. Immunol Rev 2005, 207: 145-157.

28. Shields DC, Banik NL: Upregulation of calpain activity and expression in experimental allergic encephalomyelitis: a putative role for calpain in demyelination. Brain Res 1998, 794:68-74.

29. Smerjac SM, Bizzozero OA: Cytoskeletal protein carbonylation and degradation in experimental autoimmune encephalomyelitis. J Neurochem 2008, 105:763-772.

30. Nakashima I, Fujinoki M, Fujihara K, Kawamura T, Nishimura T, Nakamura M, Itoyama $Y$ : Alteration of cystatin $C$ in the cerebrospinal fluid of multiple sclerosis. Ann Neurol 2007, 62:197-200. discussion 205

31. Carrette O, Burkhard PR, Hughes S, Hochstrasser DF, Sanchez J-C: Truncated cystatin $C$ in cerebrospiral fluid: Technical artefact or biological process? Proteomics 2005, 5:3060-3065.

32. Nicot A, Ratnakar PV, Ron Y, Chen CC, Elkabes S: Regulation of gene expression in experimental autoimmune encephalomyelitis indicates early neuronal dysfunction. Brain 2003, 1 26:398-4I2.

33. Shilov IV, Seymour SL, Patel AA, Loboda A, Tang WH, Keating SP, Hunter CL, Nuwaysir LM, Schaeffer DA: The Paragon Algorithm, a next generation search engine that uses sequence temperature values and feature probabilities to identify peptides from tandem mass spectra. Mol Cell Proteomics 2007 , 6:1638-1655

34. Hu J, Qian J, Borisov O, Pan S, Li Y, Liu T, Deng L, Wannemacher K, Kurnellas M, Patterson C, Elkabes S, Li H: Optimized proteomic 
analysis of a mouse model of cerebellar dysfunction using amine-specific isobaric tags. Proteomics 2006, 6:432I-4334.

Publish with Biomed Central and every scientist can read your work free of charge

"BioMed Central will be the most significant development for disseminating the results of biomedical research in our lifetime. " Sir Paul Nurse, Cancer Research UK

Your research papers will be:

- available free of charge to the entire biomedical community

- peer reviewed and published immediately upon acceptance

- cited in PubMed and archived on PubMed Central

- yours - you keep the copyright 